\title{
Normal polytopes and ellipsoids
}

\author{
Joseph Gubeladze \\ Department of Mathematics \\ San Francisco State University \\ 1600 Holloway Ave. \\ San Francisco, CA 94132, U.S.A. \\ soso@sfsu.edu
}

Submitted: Mar 27, 2021; Accepted: Sep 25, 2021; Published: Oct 8, 2021

(C) The author. Released under the CC BY-ND license (International 4.0).

\begin{abstract}
We show that: (1) unimodular simplices in a lattice 3-polytope cover a neighborhood of the boundary of the polytope if and only if the polytope is very ample, (2) the convex hull of lattice points in every ellipsoid in $\mathbb{R}^{3}$ has a unimodular cover, and (3) for every $d \geqslant 5$, there are ellipsoids in $\mathbb{R}^{d}$, such that the convex hulls of the lattice points in these ellipsoids are not even normal. Part (c) answers a question of Bruns, Michałek, and the author.
\end{abstract}

Mathematics Subject Classifications: 52B20, 11H06

\section{Introduction}

\subsection{Main result}

A convex polytope $P \subset \mathbb{R}^{d}$ is normal if it is lattice, i.e., has vertices in $\mathbb{Z}^{d}$, and satisfies the condition

$$
\forall c \in \mathbb{N} \quad \forall x \in(c P) \cap \mathbb{Z}^{d} \quad \exists x_{1}, \ldots, x_{c} \in P \cap \mathbb{Z}^{d} \quad x_{1}+\cdots+x_{c}=x .
$$

A necessary condition for $P$ to be normal is that the subgroup

$$
\operatorname{gp}(P):=\sum_{x, y \in P \cap \mathbb{Z}^{d}} \mathbb{Z}(x-y) \subset \mathbb{Z}^{d}
$$

must be a direct summand. Also, a face of a normal polytope is normal.

Normality is a central notion in toric geometry and combinatorial commutative algebra [7]. A weaker condition for lattice polytopes is very ample; see Section 1.2 for the definition. Normal polytopes define projectively normal embeddings of toric varieties whereas very ample polytopes correspond to normal projective varieties [3, Proposition 2.1]. 
A sufficient condition for a lattice polytope $P$ to be normal is the existence of a unimodular cover, which means that $P$ is a union of unimodular simplices. Unimodular covers play an important role in integer programming through their connection to the Integral Carathéodory Property [8, 12, 15].

There exist normal polytopes in dimensions $\geqslant 5$ without unimodular cover [6]. It is believed that all normal 3-polytopes have unimodular cover. But progress in this direction is scarce. Recent works $[4,11]$ show that all lattice 3-dimensional parallelepipeds and centrally symmetric 3-polytopes with unimodular corners have unimodular cover.

The normality of the convex hull of lattice points in an ellipsoid naturally comes up in [9]. We consider general ellipsoids, neither centered at 0 nor aligned with the coordinate axes. According to [9, Theorem 6.5(c)], the convex hull of the lattice points in any ellipsoid $E \subset \mathbb{R}^{3}$ is normal. [9, Question 7.2(b)] asks whether this result extends to higher dimensional ellipsoids.

Here we prove the following

Theorem. Let $P \subset \mathbb{R}^{3}$ be a lattice polytope, $E \subset \mathbb{R}^{d}$ an ellipsoid, and $P(E)$ the convex hull of the lattice points in E.

(a) The unimodular simplices in $P$ cover a neighborhood of the boundary $\partial P$ in $P$ if and only if $P$ is very ample.

(b) If $d=3$ then the polytope $P(E)$ is covered by unimodular simplices.

(c) For every $d \geqslant 6$, there exists $E$ such that $\operatorname{gp}(P(E))=\mathbb{Z}^{d}$ and $P(E)$ is not normal.

If in (c) we drop the condition $\operatorname{gp}(P(E))=\mathbb{Z}^{d}$, then ellipsoids $E \subset \mathbb{R}^{d}$ with $P(E)$ non-normal already exist for $d=5$; see Remark 7 .

\subsection{Preliminaries}

$\mathbb{Z}_{+}$and $\mathbb{R}_{+}$denote the sets of non-negative integers and reals, respectively.

The convex hull of a set $X \subset \mathbb{R}^{d}$ is denoted by $\operatorname{conv}(X)$. The relative interior of a convex set $X \subset \mathbb{R}^{d}$ is denoted by int $X$. The boundary of $X$ is denoted by $\partial X=X \backslash \operatorname{int} X$.

Polytopes are assumed to be convex. For a polytope $P \subset \mathbb{R}^{d}$, its vertex set is denoted by $\operatorname{vert}(P)$.

A lattice $n$-simplex $\Delta=\operatorname{conv}\left(x_{0}, \ldots, x_{n}\right) \subset \mathbb{R}^{d}$ is unimodular if $\left\{x_{1}-x_{0}, \ldots, x_{n}-x_{0}\right\}$ is a part of a basis of $\mathbb{Z}^{d}$.

A unimodular pyramid over a lattice polytope $Q$ is a lattice polytope $P=\operatorname{conv}(v, Q)$, where the point $v$ is not in the affine hull of $Q$ and the lattice height of $v$ above $Q$ inside the affine hull of $P$ equals 1 .

Cones $C$ are assumed to be pointed, rational, and finitely generated, which means $C=\mathbb{R}_{+} x_{1}+\cdots+\mathbb{R}_{+} x_{k}$, where $x_{1}, \ldots, x_{k} \in \mathbb{Z}^{d}$ and $C$ does not contain a nonzero linear subspace. For a cone $C \subset \mathbb{R}^{d}$, the smallest generating set of the additive submonoid $C \cap \mathbb{Z}^{d} \subset \mathbb{Z}^{d}$ consists of the indecomposable elements of this monoid. This is a finite set, called the Hilbert basis of $C$ and denoted by $\operatorname{Hilb}(C)$. See [7, Chapter 2] for a detailed 
discussion on Hilbert bases. For a lattice polytope $P \subset \mathbb{R}^{d}$, we have the inclusion of finite subsets of $\mathbb{Z}^{d+1}$ :

$$
\left(P \cap \mathbb{Z}^{d}, 1\right) \subset \operatorname{Hilb}\left(\mathbb{R}_{+}(P, 1)\right) .
$$

This inclusion is an equality if and only if $P$ is normal.

A lattice polytope $P$ is very ample if $\operatorname{Hilb}\left(\mathbb{R}_{+}(P-v)\right) \subset P-v$ for every vertex $v \in \operatorname{vert}(P)$. All normal polytopes are very ample, but already in dimension 3 there are very ample non-normal polytopes [7, Exercise 2.24]. For a detailed analysis of the discrepancy between the two properties see [3].

For a cone $C \subset \mathbb{R}^{d}$, we say that $C$ has a unimodular Hilbert triangulation (cover) if $C$ can be triangulated (resp., covered) by cones of the form $\mathbb{R}_{+} x_{1}+\cdots+\mathbb{R} x_{n}$, where $\left\{x_{1}, \ldots, x_{n}\right\}$ is a part of a basis of $\mathbb{Z}^{d}$ as well as of $\operatorname{Hilb}(C)$.

An ellipsoid $E \subset \mathbb{R}^{d}$ is a set of the form

$$
\left\{x \in \mathbb{R}^{d} \mid\left(l_{1}(x)-a_{1}\right)^{2}+\cdots+\left(l_{d}(x)-a_{d}\right)^{2}=1\right\} \subset \mathbb{R}^{d},
$$

where $l_{1}, \ldots, l_{d}$ is a full-rank system of real linear forms and $a_{1}, \ldots, a_{d} \in \mathbb{R}^{d}$.

For a lattice polytope $P$, the union of unimodular simplices in $P$ will be denoted by $\mathrm{U}(P)$.

\section{Unimodular covers close to the boundary}

The following result of Sebő was later rediscovered in $[1,5]$ in a refined form in the context of toric varieties.

Theorem 1. ([16]) Every 3-dimensional cone $C$ has a unimodular Hilbert triangulation.

Notice. There exist 4-dimensional cones without unimodular Hilbert triangulation [5] and it is not known whether all 4- and 5-dimensional cones have unimodular Hilbert cover. According to [6], in all dimensions $\geqslant 6$ there are cones without unimodular Hilbert cover.

If $P \subset \mathbb{R}^{3}$ is very ample, then by Theorem 1 , for every $v \in \operatorname{vert}(P)$, the cone $\mathbb{R}_{+}(P-v)$ has a unimodular Hilbert triangulation:

$$
\mathbb{R}_{+}(P-v)=\bigcup_{T(v)} C_{t},
$$

where $T(v)$ is a finite index set, depending on $v$. In particular, the following unimodular simplices form a neighborhood of $v$ in $P$ :

$$
\Delta_{v, t}=\operatorname{conv}\left(\operatorname{Hilb}\left(C_{t}\right), 0\right)+v, \quad t \in T(v) .
$$

Also, lattice polygons have unimodular triangulation [7, Corollary 2.54]. Therefore, the following lemma completes the proof of Theorem (a): 
Lemma 2. For a lattice polytope $P$ of an arbitrary dimension, the following conditions are equivalent:

(a) $\mathrm{U}(P)$ is a neighborhood of $\partial P$ within $P$;

(b) $\mathrm{U}(P)$ is a neighborhood within $P$ of every vertex of $P$ and $\partial P \subset \mathrm{U}(P)$.

Proof. The implication $(\mathrm{a}) \Longrightarrow(\mathrm{b})$ is obvious.

For the opposite implication, let:

. $x \in \partial P$;

- $F$ be the minimal face of $P$ containing $x$;

- $v \in \operatorname{vert}(F)$;

- $T_{F}$ be a unimodular cover of $F$ with $\operatorname{dim}(F)$-simplices, contained in $F$;

- $T_{v}$ be a unimodular cover of a neighbourhood of $v$ in $P$;

- $T_{v, F}$ be the sub-family of $T_{v}$, consisting of simplices that have a $\operatorname{dim}(F)$ - dimensional intersection with $F$;

- $T_{v} / F$ be the collection of faces of simplices in $T_{v, F}$, opposite to $F$ (that is, from each simplex in $T_{v, F}$ remove the $\operatorname{dim}(F)+1$ vertices that lie in $F$, so that one is left with a $(\operatorname{dim}(P)-\operatorname{dim}(F))$-simplex $)$.

Then, the collection of $\operatorname{conv}\left(T_{v} / F, T_{F}\right)$ covers a neighbourhood of $x$ in $P$ and consists of unimodular simplices.

\section{Unimodular covers inside ellipsoids}

\subsection{Proof of Theorem (b)}

The set of normal polytopes $P \subset \mathbb{R}^{d}$ carries a poset structure, where the order is generated by the elementary relation

$$
P \leqslant Q \text { if } P \subset Q \text { and } \#\left(Q \cap \mathbb{Z}^{d}\right)=\#\left(P \cap \mathbb{Z}^{d}\right)+1 .
$$

In [9] this poset is denoted by $\operatorname{NPol}(d)$. The trivial minimal elements of $\operatorname{NPol}(d)$ are the singletons from $\mathbb{Z}^{d}$. It is known that $\operatorname{NPol}(d)$ has nontrivial minimal elements for $d \geqslant 4[7$, Exercise 2.27] and the first maximal elements for $d=4,5$ were found in [9]. It is possible that $\mathrm{NPol}(d)$ has isolated elements for some $d$.

Computer searches so far have found neither maximal nor nontrivial minimal elements in $\mathrm{NPol}(3)$ [9]. The next lemma is yet another evidence that all normal 3-polytopes have unimodular cover. 
Lemma 3. Let $P$ be a normal 3-polytope. If $* \leqslant P$ in $\operatorname{NPol}(3)$ for a singleton $* \in \mathbb{Z}^{3}$ then $P=\mathrm{U}(P)$.

Proof. If $Q \leqslant P$ is an elementary relation in $\operatorname{NPol}(d)$ and $\operatorname{dim} Q<\operatorname{dim} P$ then $P$ is a unimodular pyramid over $Q$. In this case every full-dimensional unimodular simplex $\Delta \subset P$ is the unimodular pyramid over a unimodular simplex in $Q$ and with the same apex as $P$. On the other hand, lattice segments and polygons are unimodularly triangulable. Therefore, it is enough to show that a polytope $P \in \operatorname{NPol}(3)$ has a unimodular cover if there is a 3-polytope $Q \in \operatorname{NPol}(3)$, such that $Q$ has a unimodular cover and $Q \leqslant P$ is an elementary relation in $\operatorname{NPol}(3)$. Assume $\{v\}=\operatorname{vert}(P) \backslash Q$. By Theorem (a) we have the inclusion $P \backslash \mathrm{U}(P) \subset Q$. Since $Q=\mathrm{U}(Q)$ we have $P=\mathrm{U}(P)$.

Call a subset $\mathcal{E} \subset \mathbb{Z}^{d}$ ellipsoidal and a point $v \in \mathcal{E}$ extremal if there is an ellipsoid $E \subset \mathbb{R}^{d}$, such that $\mathcal{E}=\operatorname{conv}(E) \cap \mathbb{Z}^{d}$ and $v \in E$.

Lemma 4. Let $\mathcal{E} \subset \mathbb{R}^{d}$ be an ellipsoidal set. Then $\mathcal{E}$ has an extremal point and $\mathcal{E} \backslash\{v\}$ is also ellipsoidal for every extremal point $v \in \mathcal{E}$.

Proof. Let $\mathcal{E}=\operatorname{conv}(E) \cap \mathbb{Z}^{d}$ for an ellipsoid $E \subset \mathbb{R}^{d}$. Applying an appropriate homothetic contraction, centered at the center of $E$, we can always achieve $\mathcal{E} \cap E \neq \emptyset$. In particular, $\mathcal{E}$ has an extremal point. For $v \in \mathcal{E} \cap E$, after changing $E$ to its homothetic image with factor $(1+\varepsilon)$ and centered at $v$, where $\varepsilon$ is a sufficiently small positive real number, we can further assume $\mathcal{E} \cap E=\{v\}$. Finally, applying a parallel translation to $E$ by $\delta(z-v)$, where $z$ is the center of $E$ and $\delta>0$ is a sufficiently small real number, we achieve $\operatorname{conv}(E) \cap \mathbb{Z}^{d}=\mathcal{E} \backslash\{v\}$.

Next we complete the proof of Theorem (b). It follows from Lemma 3.2 that, for any natural number $d$ and an ellipsoidal set $\mathcal{E} \subset \mathbb{Z}^{d}$, there is a descending sequence of ellipsoidal sets of the form

$$
\mathcal{E}=\mathcal{E}_{k} \supset \mathcal{E}_{k-1} \supset \cdots \supset \mathcal{E}_{1} \text {, with } \# \mathcal{E}_{i}=i \text { for } i=1, \ldots, k .
$$

By $[9$, Theorem $6.5(\mathrm{c})]$, for $d=3$, the $\operatorname{conv}\left(\mathcal{E}_{i}\right)$ are normal polytopes. Therefore, $* \leqslant \operatorname{conv}(\mathcal{E})$ in $\operatorname{NPol}(3)$ for some $* \in \mathbb{Z}^{3}$. Thus Lemma 3 applies.

\subsection{Alternative algorithmic proof in symmetric case}

For the ellipsoids $E$ with center in $\frac{1}{2} \mathbb{Z}^{3}$, there is a different proof of Theorem (b). It yields a simple algorithm for constructing a unimodular cover of $P(E)$.

Instead of Theorem 1 and [9, Theorem 6.5] this approach uses Johnson's 1916 Circle Theorem $[13,14]$. We only need Johnson's theorem to derive the following fact, which does not extend to higher dimensions: for any lattice $\Lambda \subset \mathbb{R}^{2}$ and any ellipse $E^{\prime} \subset \mathbb{R}^{2}$, such that $\operatorname{conv}\left(E^{\prime}\right)$ contains a triangle with vertices in $\Lambda$, every parallel translate $\operatorname{conv}\left(E^{\prime}\right)+v$, where $v \in \mathbb{R}^{2}$, meets $\Lambda$.

Assume an ellipsoid $E \subset \mathbb{R}^{3}$ has center in $\frac{1}{2} \mathbb{Z}^{3}$ and $\operatorname{dim}(P(E))=3$ (notation as in the theorem). Assume $\mathrm{U}(P(E)) \varsubsetneqq P(E)$. Because $\partial P(E)$ is triangulated by unimodular 
triangles, there is a unimodular triangle $T \subset P(E)$, not necessarily in $\partial P(E)$, and a point $x \in \operatorname{int} T$, such that the points in $[0, x]$, sufficiently close to $x$, are not in $\mathrm{U}(P(E))$. For the plane, parallel to $T$ on lattice height 1 above $T$ and on the same side as 0 , the intersection $E^{\prime}=\operatorname{conv}(E) \cap H$ is at least as large as the intersection of $\operatorname{conv}(E)$ with the affine hull of $T$ : a consequence of the fact that $P(E) \cap \mathbb{Z}^{3}$ is symmetric relative to the center of $E$. The mentioned consequence of Johnson's theorem implies that $\operatorname{conv}\left(E^{\prime}\right)$ contains a point $z \in \mathbb{Z}^{3}$. In particular, all points in $[x, 0]$, sufficiently close to $x$ are in the unimodular simplex $\operatorname{conv}(T, z) \subset P(E)$, a contradiction.

\section{High dimensional ellipsoids}

For a lattice $\Lambda \subset \mathbb{R}^{d}$, define a $\Lambda$-polytope as a polytope $P \subset \mathbb{R}^{d}$ with $\operatorname{vert}(P) \subset \Lambda$. Using $\Lambda$ as the lattice of reference instead of $\mathbb{Z}^{d}$, one similarly defines $\Lambda$-normal polytopes and $\Lambda$-ellipsoidal sets.

Consider the lattice $\Lambda(d)=\mathbb{Z}^{d}+\mathbb{Z}\left(\frac{1}{2}, \ldots, \frac{1}{2}\right) \subset \mathbb{R}^{d}$. We have $\left[\mathbb{Z}^{d}: \Lambda(d)\right]=2$. Consider the $\Lambda(d)$-polytope $P(d)=\operatorname{conv}(\mathrm{B}(d) \cap \Lambda(d))$, where $\mathrm{B}(d)=\left\{\left(\xi_{1}, \ldots, \xi_{d}\right) \mid \sum_{i=1}^{d}\left(\xi_{i}-\right.\right.$ $\left.\left.\frac{1}{2}\right)^{2} \leqslant \frac{d}{4}\right\} \subset \mathbb{R}^{d}$, i.e., $\partial(\mathrm{B}(d))$ is the circumscribed sphere for the cube $[0,1]^{d}$.

Consider the $d$-dimensional $\Lambda(d)$-polytope and the $(d-1)$-dimensional $\Lambda(d)$-simplex:

$$
\begin{aligned}
& Q(d)=\operatorname{conv}\left((P(d) \cap \Lambda(d)) \backslash\left\{\mathbf{e}_{1}+\cdots+\mathbf{e}_{d}\right\}\right), \\
& \Delta(d-1)=\operatorname{conv}\left(\mathbf{e}_{1}+\cdots+\mathbf{e}_{i-1}+\mathbf{e}_{i+1}+\cdots+\mathbf{e}_{d} \mid i=1, \ldots, d\right),
\end{aligned}
$$

where $\mathbf{e}_{1}, \ldots, \mathbf{e}_{d} \in \mathbb{R}^{d}$ are the standard basic vectors.

Notice. Although $P(d) \cap \mathbb{Z}^{d}=\{0,1\}^{d}$ for all $d$, yet $[0,1]^{d} \varsubsetneqq P(d)$ for all $d \geqslant 4$. In fact, $\left(\frac{1}{2}, \ldots, \frac{1}{2}\right)+k \mathbf{e}_{i} \in P(d) \cap \Lambda(d)$ for $1 \leqslant i \leqslant d$ and $-\left\lceil\frac{\sqrt{d}}{2}\right\rceil \leqslant k \leqslant\left\lfloor\frac{\sqrt{d}}{2}\right\rfloor$.

Lemma 5. If $d \geqslant 5$ then $\Delta(d-1)$ is a facet of $Q(d)$ and $\Delta(d-1) \cap \Lambda(d)=\operatorname{vert}(\Delta(d-1))$.

Proof. Assume $x=\left(\xi_{1}, \ldots, \xi_{d}\right) \in P(d) \cap \Lambda(d)$ satisfies $\xi_{1}+\cdots+\xi_{d} \geqslant d-1$. We claim that there are only two possibilities: either $x=\mathbf{e}_{1}+\cdots+\mathbf{e}_{d}$ or $x=\mathbf{e}_{1}+\cdots+\mathbf{e}_{i-1}+\mathbf{e}_{i+1}+\cdots+\mathbf{e}_{d}$ for some index $i$. Since $P(d) \cap \mathbb{Z}^{d}=\{0,1\}^{d}$, only the case $x \in\left(\frac{1}{2}, \ldots, \frac{1}{2}\right)+\mathbb{Z}^{d}$ needs to be ruled out. Assume $\xi_{i}=\frac{1}{2}+a_{i}$ for some integers $a_{i}$, where $i=1, \ldots, d$. Then we have the inequalities

$$
\sum_{i=1}^{d} a_{i}^{2} \leqslant \frac{d}{4} \quad \text { and } \quad \sum_{i=1}^{d} a_{i} \geqslant \frac{d}{2}-1 .
$$

Since the $a_{i}$ are integers we have $\frac{d}{4} \geqslant \frac{d}{2}-1$, a contradiction because $d \geqslant 5$.

Lemma 6. For every even natural number $d \geqslant 6$, there exists a point in $\left(\frac{d}{2} \cdot Q(d)\right) \cap \Lambda(d)$ which does not have a representation of the form $x_{1}+\cdots+x_{\frac{d}{2}}$ with $x_{1}, \ldots, x_{\frac{d}{2}} \in Q(d) \cap \Lambda(d)$. In particular, $Q(d)$ is not $\Lambda(d)$-normal. 
Proof. Consider the baricenter $\beta(d)=\frac{d-1}{d} \cdot\left(\mathbf{e}_{1}+\cdots+\mathbf{e}_{d}\right)$ of $\Delta(d-1)$. The point $\frac{d}{2} \cdot \beta(d)$ is the baricenter of the dilated simplex $\frac{d}{2} \cdot \Delta(d-1)$ and, simultaneously, a point in $\Lambda(d)$. Assume $\frac{d}{2} \cdot \beta=x_{1}+\cdots+x_{\frac{d}{2}}$ for some $x_{1}, \ldots, x_{\frac{d}{2}} \in Q(d) \cap \Lambda(d)$. Lemma 5 implies $x_{1}, \ldots, x_{\frac{d}{2}} \in \Delta(d-1) \cap \Lambda(d)^{2}=\operatorname{vert}(\Delta(d-1))$. But this is not possible because the dilated $(d-1)$-simplex $c \Delta(d-1)$ has an interior point of the form $z_{1}+\cdots+z_{c}$ with $z_{1}, \ldots, z_{c} \in \operatorname{vert}(\Delta(d-1))$ only if $c \geqslant d$.

Proof of Theorem (c). Since $\mathbf{e}_{1}, \ldots, \mathbf{e}_{d},\left(\frac{1}{2}, \ldots, \frac{1}{2}\right) \in Q(d)$ we have the equality $g \mathrm{p}(Q(d))=$ $\Lambda(d)$. By Lemmas 4 and 5, the set $Q(d) \cap \Lambda(d)$ is $\Lambda(d)$-ellipsoidal for $d \geqslant 5$. By applying a linear transformation, mapping $\Lambda(d)$ isomorphically to $\mathbb{Z}^{d}$, Lemma 6 already implies Theorem (c) for $d$ even.

One involves all dimensions $d \geqslant 6$ by observing that (i) if $\mathcal{E} \subset \mathbb{R}^{d}$ is an ellipsoidal set then $\mathcal{E} \times\{0,1\} \subset \mathbb{R}^{d+1}$ is also ellipsoidal and (ii) the normality of $\operatorname{conv}(\mathcal{E} \times\{0,1\})$ implies that of $\operatorname{conv}(\mathcal{E})$. While (ii) is straightforward, for (i) one applies an appropriate affine transformation to achieve $\mathcal{E}=\operatorname{conv}\left(S^{d-1}\right) \cap \Lambda$, where $S^{d-1} \subset \mathbb{R}^{d}$ is the unit sphere, and $\Lambda \subset \mathbb{R}^{d}$ is a shifted lattice. In this case the ellipsoid $E=\left\{\left(\xi_{1}, \ldots, \xi_{d}\right) \mid \frac{\xi_{1}^{2}}{a^{2}}+\right.$ $\left.\cdots+\frac{\xi_{d-1}^{2}}{a^{2}}+\frac{\xi_{d}^{2}}{a^{2}}+\frac{\left(\xi_{d+1}-\frac{1}{2}\right)^{2}}{b^{2}}=1\right\} \subset \mathbb{R}^{d+1}$ with $b>\frac{1}{2}$ and $a=\frac{2 b}{\sqrt{4 b^{2}-1}}$, is within the $\left(b-\frac{1}{2}\right)$-neighborhood of the region of $\mathbb{R}^{d+1}$ between the hyperplanes $\left(\mathbb{R}^{d}, 0\right)$ and $\left(\mathbb{R}^{d}, 1\right)$ and satisfies the following conditions: $E \cap\left(\mathbb{R}^{d}, 0\right)=\left(S^{d-1}, 0\right)$ and $E \cap\left(\mathbb{R}^{d}, 1\right)=\left(S^{d-1}, 1\right)$. In particular, when $\frac{1}{2}<b<\frac{3}{2}$ we have $\operatorname{conv}(E) \cap(\Lambda \times \mathbb{Z})=\mathcal{E} \times\{0,1\}$.

Remark 7 . The definition of a normal polytope in the introduction is stronger than the one in [7, Definition 2.59]: the former is equivalent to the notion of an integrally closed polytope, whereas 'normal' in the sense of [7] is equivalent to $\mathrm{gp}(P)$-normal. Examples of $\operatorname{gp}(P)$-normal polytopes, which are not normal, are lattice non-unimodular simplices, whose only lattice points are the vertices. Lemma 5 and the proof of Lemma 6 show that the 5 -simplex $\Delta(5)$ is not $\Lambda(6)$-unimodular. Applying an appropriate affine transformation we obtain a lattice non-unimodular simplices $\Delta^{\prime} \subset \mathbb{R}^{5}$ with $\operatorname{vert}\left(\Delta^{\prime}\right)$ ellipsoidal. Such examples in $\mathbb{R}^{5}$ have been known sine the 1970s: a construction of Voronoi [2] yields a lattice $\Lambda \subset \mathbb{R}^{5}$ and a 5 -simplex $\Delta \subset \mathbb{R}^{5}$ of $\Lambda$-multiplicity 2 , whose circumscribed sphere does not contain points of $\Lambda$ inside except vert $(\Delta)$.

We do not know whether there are ellipsoidal subsets $\mathcal{E} \subset \mathbb{R}^{5}$ with conv $(\mathcal{E})$ non-normal and $\operatorname{gp}(\operatorname{conv}(\mathcal{E}))=\mathbb{Z}^{5}$. For instance, $Q(5)$ is $\Lambda(5)$-normal, as checked by Normaliz [10].

\section{Acknowledgments}

We thank the referees for the streamlined version of the original proof of Theorem (a), bringing [2] to our attention, and spotting several inaccuracies. 


\section{References}

[1] Stefano Aguzzoli and Daniele Mundici. An algorithmic desingularization of 3dimensional toric varieties. Tohoku Math. J. (2), 46(4):557-572, 1994. DOI: 10.2748/tmj/1178225680.

[2] E. P. Baranovskiǐ. Volumes of $L$-simplexes of five-dimensional lattices. Mat. Zametki, 13:771-782, 1973. DOI: 10.1007/BF01147478.

[3] Matthias Beck, Jessica Delgado, Joseph Gubeladze, and Mateusz Michałek. Very ample and Koszul segmental fibrations. J. Algebraic Combin., 42(1):165-182, 2015. DOI: $10.1007 / \mathrm{s} 10801-014-0577-7$.

[4] Matthias Beck, Christian Haase, Akihiro Higashitani, Johannes Hofscheier, Katharina Jochemko, Lukas Katthän, and Mateusz Michałek. Smooth centrally symmetric polytopes in dimension 3 are IDP. Ann. Comb., 23(2):255-262, 2019. DOI: 10.1007/s00026-019-00418-x.

[5] Catherine Bouvier and Gérard Gonzalez-Sprinberg. Système générateur minimal, diviseurs essentiels et $G$-désingularisations de variétés toriques. Tohoku Math. J. (2), 47(1):125-149, 1995. DOI: 10.2748/tmj/1178225640.

[6] Winfried Bruns and Joseph Gubeladze. Normality and covering properties of affine semigroups. J. Reine Angew. Math., 510:161-178, 1999. DOI: $10.1515 / \operatorname{crll} .1999 .044$.

[7] Winfried Bruns and Joseph Gubeladze. Polytopes, rings, and K-theory. Springer Monographs in Mathematics. Springer-Verlag, New York, 2009. DOI: $10.1007 / \mathrm{b} 105283$.

[8] Winfried Bruns, Joseph Gubeladze, Martin Henk, Alexander Martin, and Robert Weismantel. A counterexample to an integer analogue of Carathéodory's theorem. J. Reine Angew. Math., 510:179-185, 1999. DOI: 10.1515/crll.1999.045.

[9] Winfried Bruns, Joseph Gubeladze, and Mateusz Michałek. Quantum Jumps of Normal Polytopes. Discrete Comput. Geom., 56(1):181-215, 2016. DOI: 10.1007/s00454-016-9773-7.

[10] Winfried Bruns, Bogdan Ichim, Tim Römer, and Christof Söger. Normaliz. Available from http://www.math.uos.de/normaliz/.

[11] Giulia Codenotti and Francisco Santos. Unimodular covers of 3-dimensional parallepipeds and Caley sums. Preprint, arXiv:1907.12312.

[12] William Cook, Jean Fonlupt, and Alexander Schrijver. An integer analogue of Carathéodory's theorem. J. Combin. Theory Ser. B, 40(1):63-70, 1986. DOI: 10.1016/0095-8956(86)90064-X.

[13] Frank Jackson and Eric W. Weisstein. Johnson circles. https://mathworld. wolfram.com/JohnsonCircles.html.

[14] Roger A. Johnson. A Circle Theorem. Amer. Math. Monthly, 23(5):161-162, 1916. DOI: $10.1080 / 00029890.1916 .11998200$. 
[15] Alexander Schrijver. Theory of linear and integer programming. Wiley-Interscience Series in Discrete Mathematics. John Wiley \& Sons, Ltd., Chichester, 1986. A WileyInterscience Publication.

[16] Andras Sebő. Hilbert bases, Carathéodory's theorem and combinatorial optimization. Proc. of the IPCO conference (Waterloo, Canada), pages 431-455, 1990. 